\title{
Editorial: Flame-Retardant Polymeric Materials and Polymer Composites
}

\author{
Yongqian Shi ${ }^{1 *}$, Bin $\mathrm{Yu}^{2}$, Xin Wang ${ }^{3}$ and Anthony Chun Yin Yuen ${ }^{4}$ \\ ${ }^{1}$ College of Environment and Resources, Fuzhou University, Fuzhou, China, ${ }^{2}$ Centre for Future Materials, University of Southern \\ Queensland Toowoomba, Toowoomba, QLD, Australia, ${ }^{3}$ State Key Laboratory of Fire Science, University of Science and \\ Technology of China, Hefei, China, ${ }^{4}$ School of Mechanical and Manufacturing Engineering, University of New South Wales, \\ Kensington, NSW, Australia
}

Keywords: polymer composites, flame retardancy, smoke toxicity, fire simulation, sustainability

Editorial on the Research Topic

Flame-Retardant Polymeric Materials and Polymer Composites

\section{INTRODUCTION}

Lightweight polymeric materials have been widely applied in modern society. However, many of these polymeric materials are highly flammable, causing great fire risks to people's lives and belongings. The fire hazards associated with these flammable polymeric materials have been regarded as a major issue to be addressed. These flammable polymeric materials have been identified as the root cause of many severe fire incidents, which have given rise to over 40,000 deaths worldwide and total fire losses accounting for around $1 \%$ of the gross domestic product (GDP) of the country. Therefore, the minimization of the fire risk of these flammable polymeric materials has become a compulsory requirement for their practical application in industry. The development of environmentally benign flame-retardant polymers/composites represents one effective strategy to address the fire issues associated with these flammable polymers.

This research topic, Flame-Retardant Polymeric Materials and Flame-Retardant Polymer Composites (FRPMs and FRPCs), aims to feature the latest technological and scientific advances and future opportunities and challenges on flame-retardant materials. We also aim to broaden and deepen the scientific and technological knowledge with the most recent advances in the preparation, performance, and application of FRPMs and FRPCs with respect to the concept of long-term environmental, economic, and social sustainability. This research topic will be of great value to engineers, scientists, and decision-makers who are working in academia, industry, and government sectors. Also, it will greatly contribute to catalyzing the discovery and development of FRPMs and FRPCs while highlighting their associated challenges in sustainable development. As depicted in Figure 1, the cutting-edge flame retardant technologies utilized for polymer composites can be subdivided into three main categories: 1) char-enhancing, 2) self-extinguishing and 3) bio-inspiring.

This series includes eight research articles that cover a wide range of flame retardant polymer materials, including epoxy resin (Kong et al., 2020; Yi et al., 2020), polypropylene (Liu et al., 2020), polystyrene (Shi et al., 2020), polyurethane (Hu et al., 2021), polyvinyl alcohol (Wang et al., 2020) and flame retardants based fire-fighting foams (Li et al., 2021a; Li et al., 2021b). 
A

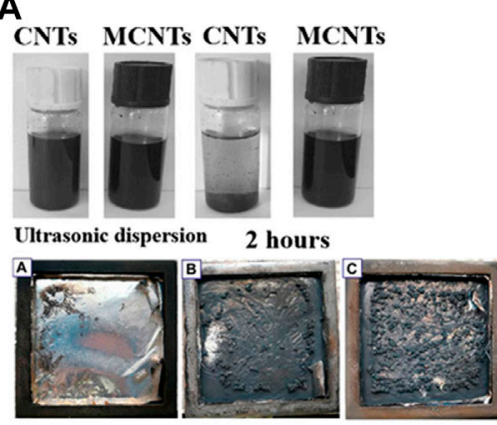

Carbon-based char enhancer after Shi et al 2020

$\mathbf{C}_{A}$

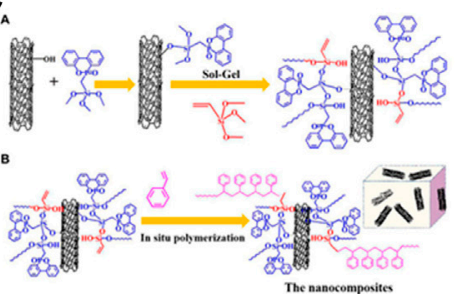

Fabrication process of functionalized CNTs with DOPO after Shi et al. 2020
Flame-Retardant Polymeric Materials and Polymer Composites
B
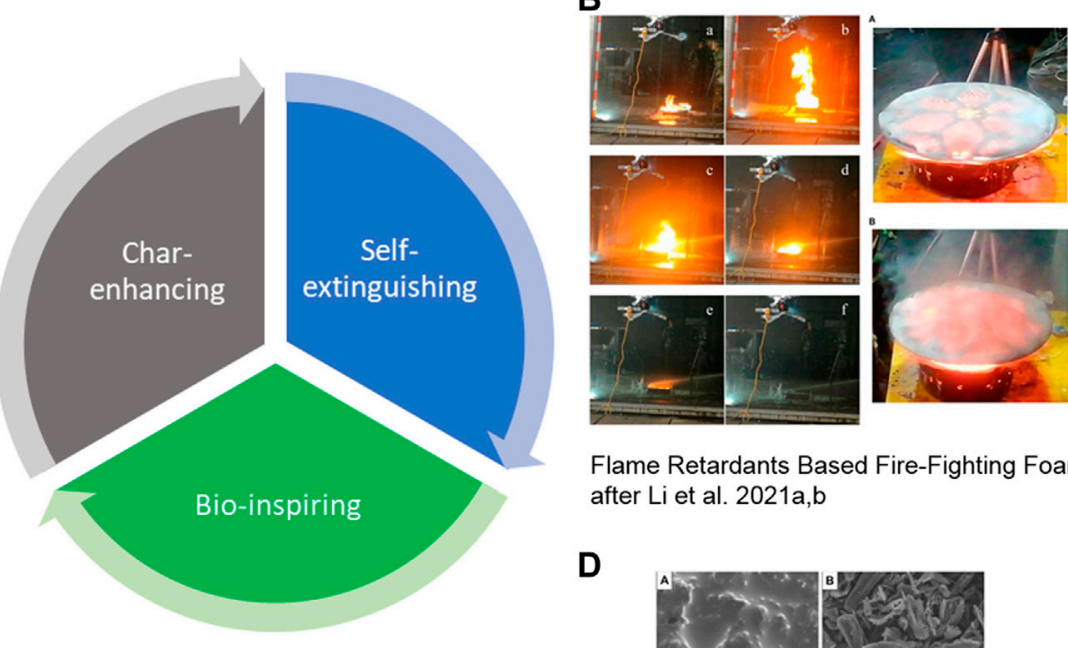

Flame Retardants Based Fire-Fighting Foam after Li et al. 2021a,b

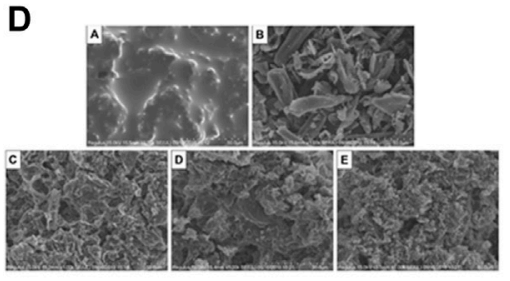

Morphology of Bamboo Flour modified composites after Liu et al. 2021

FIGURE 1 | Advancement and driving mechanisms of flame-retardant technologies for polymer composites. Images in (A), (B), (C) and (D) are adapted from Shi et al. (2020), Li et al. (2021a), Li et al. (2021b), and Liu et al. (2021) respectively, published under the terms of Creative Commons Attribution 4.0 (CC BY 4.0).

Yi et al. (2020) presented a Spiro-phosphorus (P)-based reactive flame-retardant epoxy resin curing agent. The synthesized epoxy thermosets exhibit higher carbon residue yield with significant improvements to flame retardancy and mechanical properties.

Kong et al. (2020) studied the effects of graphene oxidecobalt-nickel phosphate $\left(\mathrm{GO}-\mathrm{NiCoPO}_{3}\right)$ on the flame retardancy of epoxy resin. The results showed $\mathrm{GO}-\mathrm{NiCoPO}$ significantly enhanced char formation to reduce heat transfer, inhibit combustion, and improve the thermal stability of composites.

Liu et al. (2020) presented a flame retardant polypropylene composite via melt blending, incorporating ammonium polyphosphate (APP) and bamboo flour (BF). The effects of BF mechanical properties, crystallization behavior, thermal degradation, flame retardancy, and especially the smoke suppression effect of PP/APP composite materials were studied.

Shi et al. (2020) synthesized DOPO, and silicon-containing agents modified multiwalled carbon nanotubes (MCNTs) and investigated the application as a reinforcer for thermal and flame retardant properties of polystyrene nanocomposites.

$\mathrm{Hu}$ et al. (2021) presented flame retarded rigid polyurethane foams composites incorporating aluminium diethylphosphinate (ADP) combined with expanded graphite (EG) to form a synergistic flame retarded system. The effects of ADP and EG on the structure, thermal conductivity, thermal stability, and flame retardant performance of RPUF was investigated.

Wang et al. (2020) studied ternary h-BN@PDA@TiO2 hybrid nanoparticle as functional fillers for PVA nanocomposites. The results showed that the hybrid particles could significantly improve the thermal conductivity and flame retardant performance of the PVA composites and effectively inhibit toxic gases emissions such as combustible pyrolysis products and $\mathrm{CO}$.

Li et al. (2021a) presented the preparation and fire suppression performance of mixing nano magnesium hydroxide particles and water-soluble flame retardant 8124 as an aqueous film forming fire extinguishing agent (AFFF). The suppression agent was applied on a $30 \mathrm{~cm}$ circular gasoline fire, and performance was investigated by analyzing the $\mathrm{CO}$ concentration and gas temperature.

Li et al. (2021b) also studied the addition of Talc into AFFF extinguishing agent. The fire resistance and fire extinguishing properties of the composite foam were studied. The network structure of composite foam was important to the improved stability of the foam, and the Talc powder formed a dense layer covering the oil surface, which effectively isolated the oil from the air.

In combination, these complementary contributions provide a body of knowledge in the field of Flame-Retardant Polymeric Materials and Flame-Retardant Polymer Composites, hence the apt name of this exciting publication. 


\section{AUTHOR CONTRIBUTIONS}

All authors listed have made a substantial, direct, and intellectual contribution to the work and approved it for publication. YS writes the manuscript; $\mathrm{BY}, \mathrm{XW}$, and $\mathrm{AY}$ have revised the manuscript.

\section{FUNDING}

This work is financially supported by the National Natural Science Foundation of China (Grant No. 51803031) and

\section{REFERENCES}

Hu, Y., Zhou, Z., Li, S., Yang, D., Zhang, S., and Hou, Y. (2021). Flame Retarded Rigid Polyurethane Foams Composites Modified by Aluminum Diethylphosphinate and Expanded Graphite. Front. Mater. 7, 471. doi:10. 3389/fmats.2020.629284

Kong, Q., Zhang, C., Zheng, G., Zhang, M., Zhou, T., and Zhang, J. (2020). Effect of Graphene Oxide-Modified Cobalt Nickel Phosphate on Flame Retardancy of Epoxy Resin. Front. Mater. 7, 316. doi:10.3389/fmats.2020.588518

Li, X., Guo, R., and Qian, X. (2021a). Preparation and Absorption Carbon Monoxide Properties of a Novel Flame Retardants Based Fire-Fighting Foam. Front. Mater. 8, 48. doi:10.3389/fmats.2021.646509

Li, X., Guo, R., and Qian, X. (2021b). Study on Flame Retardancy and Mechanism of Talc Composite Foams. Front. Mater. 8, 61. doi:10.3389/fmats.2021.661906

Liu, Y., Li, H., Chen, Q., Luo, F., and Cao, C. (2020). Effect of Bamboo Flour on Flame Retardancy and Smoke Suppression of Polypropylene/Ammonium Polyphosphate Composites. Front. Mater. 7, 311. doi:10.3389/fmats.2020. 574924

Shi, C., Qian, X., Jing, J., and Che, H. (2020). Functionalized CNTs with DOPO and Silicon Containing Agents: Effective Reinforcer for Thermal and Flame
Australian Research Council Industrial Transformation Training Center (ARC IC170100032).

\section{ACKNOWLEDGMENTS}

This issue would not have been possible without the contributions of talented authors, hardworking and professional reviewers, and the dedicated editorial team of Frontiers in Materials. Congratulations to all authors who have contributed to this special issue. In addition, we would like to express our sincere gratitude to all reviewers and the editorial team of Frontiers in Materials.

Retardant Properties of Polystyrene Nanocomposites. Front. Chem. 8, 627642. doi: $10.3389 /$ fchem.2020.627642

Wang, X., Hu, W., and Hu, Y. (2020). Polydopamine-Bridged Synthesis of Ternary h-BN@PDA@TiO2 as Nanoenhancers for Thermal Conductivity and Flame Retardant of Polyvinyl Alcohol. Front. Chem. 8, 587474. doi:10.3389/fchem. 2020.587474

Yi, L., Huang, Z., Cao, Y., and Peng, Y. (2020). Synthesis of a Novel Spiro Phosphorus-Nitrogen Concerted Reactive Flame-Retardant Curing Agent and its Application in Epoxy Resin. Front. Mater. 7, 293. doi:10.3389/fmats. 2020.00293

Conflict of Interest: The authors declare that the research was conducted in the absence of any commercial or financial relationships that could be construed as a potential conflict of interest.

Copyright (c) 2021 Shi, Yu, Wang and Yuen. This is an open-access article distributed under the terms of the Creative Commons Attribution License (CC BY). The use, distribution or reproduction in other forums is permitted, provided the original author(s) and the copyright owner(s) are credited and that the original publication in this journal is cited, in accordance with accepted academic practice. No use, distribution or reproduction is permitted which does not comply with these terms. 